\title{
Robust technique for estimating the bearings of cyclostationary signals ${ }^{\text {th }}$
}

\author{
Ju-Hong Lee*, Yung-Ting Lee \\ Department of Electrical Engineering, National Taiwan University, Room 517, Building 2, Taipei 106, Taiwan
}

Received 15 June 2001; received in revised form 30 November 2002

\begin{abstract}
This paper deals with the problem of direction finding using cyclostationary signals in the presence of cycle frequency error (CFE). By exploiting the signal cyclostationarity, cyclic MUSIC and conjugate cyclic MUSIC presented in Gardner (Cyclostationarity in Communications and Signal Processing, New York, 1994; Proc. IEEE 76 (1988) 845) have been shown to be effective for signal-selective direction finding. However, they suffer from severe performance degradation even if there is a small error in the cycle frequency of the signals of interest. In this paper, we first evaluate the performance of conjugate cyclic MUSIC when CFE exists. An analytical formula is derived to show the behavior of the performance degradation due to CFE. By exploiting the eigenstructure of the conjugate cyclic correlation matrix of the received array data, we develop a technique including a method for estimating the cycle frequency of the SOI and an efficient method in conjunction with a subspace-fitting algorithm to achieve robust direction finding against CFE. Several simulation examples confirming the theoretical analysis and showing the effectiveness of the proposed technique are also presented.
\end{abstract}

(C) 2003 Elsevier Science B.V. All rights reserved.

Keywords: Cyclostationarity; Cycle frequency error; Array processing; Bearing estimation

\section{Introduction}

Array signal processing has been a topic of great interest, especially in the area of direction finding (DF) which has many applications such as radar, sonar and seismology. The objective of DF is to estimate the directions of arrival (DOAs) of the signals received by an antenna array. The MUSIC (multiple signal classification) algorithm of [12] exploits the signal or noise subspace of the autocorrelation matrix of the received array data to obtain the DOA estimation based on the following assumptions: (1) The autocorrelation matrix of noise is proportional to the identity matrix. (2) The number of signals arriving at the array is less than the number of sensor elements. (3) The columns of the array response matrix are linearly independent. However, the knowledge of noise autocorrelation matrix is often not available and not all of the DOAs of signals are of interest in many applications.

\footnotetext{
This work was supported by the National Science Council under Grant NSC89-2213-E002-133. Submission date: June 11, 2001. Revision Date: December 2, 2002.

* Corresponding author. Fax: 886-2-23638247.

E-mail address: juhong@cc.ee.ntu.edu.tw (J.-H. Lee).
} 
Cyclostationarity $[3,5,7]$ which is a statistical property corresponds to the underlying periodicity arising from carrier frequencies or baud rates. Many cyclostationarity-exploiting methods exhibit signal-selectivity in certain types of environments when enough data samples are available. Cyclic MUSIC and conjugate cyclic MUSIC presented in [4,6] accommodate multiple signals having the same cycle frequency by using a subspace-fitting approach similar to MUSIC. However, the actual cycle frequency may not be exactly known in some applications due to, for example, the phenomenon of Doppler shift. Thus, it is worth evaluating the performance of cyclic MUSIC and conjugate cyclic MUSIC under cycle frequency error (CFE) and developing techniques to minimize the resulting performance degradation due to CFE. Some previous work considering the problem has been reported in [1,9-11]. A statistical analysis presented in [9,11] shows the cycle leakage through a sinc function due to finite data collection. On the other hand, two approaches, namely, multi-cyclic MUSIC and adaptive- $\alpha$ cyclic MUSIC have been presented in [10] to cope with the performance degradation due to CFE. However, they cannot tackle the problem effectively. Variations of the adaptive- $\alpha$ cyclic MUSIC are investigated in [1]. Based on the concept of subintervals, only the cyclic correlation matrices of the subintervals are required to reduce the memory requirements and the sensitivity of cyclic MUSIC to CFE. However, the techniques developed in [1] limit the cycle frequency coverage due to the use of subintervals. Recently, several works concerning the estimation of cycle frequency error (carrier frequency offset) have been presented in $[8,13]$. Basically, these methods are developed based on the exploitation of the cyclostationarity property of the received signal data. The estimated carrier frequency offset is then used for solving some problems present in communication channels.

In this paper, we first evaluate the performance of conjugate cyclic MUSIC when the CFE regarding the SOIs exists. The theoretical results show that severe deviations between the estimated and actual DOAs appear periodically as the number of data snapshots used increases. This is the effect of cycle leakage through a sinc window due to finite data samples. Using the inherent eigenstructure properties possessed by the conjugate cyclic correlation matrix of the received array data, we present a method to estimate the cycle frequency of the SOI. Based on this estimate, an efficient method in conjunction with the fast subspace decomposition (FSD) algorithm [14] is developed to achieve robust direction finding. A previous work concerning the subspace tracking similar to the FSD algorithm has been presented in [2]. Simulation results show that the proposed technique outperforms conjugate cyclic MUSIC in the presence of CFE and provides satisfactory performance no matter whether the CFE exists or not.

This paper is organized as follows: In Section 2, we briefly describe the signal cyclostationarity and conjugate cyclic MUSIC. The performance of conjugate cyclic MUSIC in the presence of CFE is evaluated in Section 3. To tackle the effect of CFE, Section 4 presents a method for estimating the actual cycle frequency and an efficient method in conjunction with the FSD algorithm of [14] to achieve robust direction finding. Section 5 presents several simulation examples for confirming the theoretical analysis and showing the effectiveness of the proposed technique. Finally, we conclude this paper in Section 6.

\section{Direction finding using cyclostationary signals}

\subsection{Signal cyclostationarity}

For a signal $s(t)$, the conjugate cyclic correlation function is defined as

$$
r_{s s^{*}}(f, \tau)=\left\langle s(t+\tau / 2) s(t-\tau / 2) \mathrm{e}^{-\mathrm{j} 2 \pi f t}\right\rangle_{\infty},
$$

where $\langle\cdot\rangle_{\infty}$ denotes the average over an infinite time interval. $s(t)$ is said to be cyclostationary if $r_{s s^{*}}(f, \tau)$ does not equal zero at some time delay $\tau$ and cycle frequency $f \neq 0$. Many modulated signals exhibit cyclostationarity with cycle frequency equal to twice the carrier frequency or multiples of the baud rate or 
combinations of these. Let $\mathbf{x}(t)$ denote the data vector received by an antenna array. The conjugate cyclic correlation matrix is given by

$$
\mathbf{R}_{x x^{*}}(f, \tau)=\left\langle\mathbf{x}(t+\tau / 2) \mathbf{x}^{\mathrm{T}}(t-\tau / 2) \mathrm{e}^{-\mathrm{j} 2 \pi f t}\right\rangle_{\infty},
$$

where the superscript " $\mathrm{T}$ " denotes the transpose.

\subsection{Conjugate cyclic MUSIC (CC-MUSIC)}

Consider an $M$-element array excited by $d$ SOIs, $J$ SNOIs, and noise. The received data vector $\mathbf{x}(t)$ is then given by

$$
\mathbf{x}(t)=\sum_{m=1}^{d} s_{m}(t) \mathbf{a}\left(\theta_{m}\right)+\sum_{l=1}^{J} i_{l}(t) \mathbf{a}\left(\phi_{l}\right)+\mathbf{n}(t),
$$

where $s_{m}(t)$ and $i_{l}(t)$ denote the waveforms of the $m$ th SOI and the $l$ th SNOI, $\mathbf{a}\left(\theta_{m}\right)$ and $\mathbf{a}\left(\phi_{l}\right)$ the directional vectors of $s_{m}(t)$ and $i_{l}(t)$, and $\mathbf{n}(t)$ the noise, respectively. Assume that the $m$ th SOI and the $l$ th SNOI have the cycle frequencies equal to $\alpha$ and $\beta_{l}$, respectively. Moreover, the SOIs, SNOIs, and noise are temporally uncorrelated with each other.

Let $\hat{\mathbf{R}}_{x x^{*}}(\alpha, \tau, N)$ represent the sample conjugate cyclic correlation matrix computed by using $N$ data snapshots. Then, CC-MUSIC is implemented as follows [6, pp. 214-215]:

First, compute $\hat{\mathbf{R}}_{x x^{*}}(\alpha, \tau, N)=1 / N \sum_{i=1}^{N} \mathbf{x}(i) \mathbf{x}^{\mathrm{T}}(i-\tau) \mathrm{e}^{-\mathrm{j} 2 \pi \alpha i T_{s}}$, where $\mathbf{x}(i)$ denotes the vector of $\mathbf{x}(t)$ sampled at the time instant $i T_{s}$ and $T_{s}$ is the sampling interval. Then, perform the singular value decomposition (SVD) on $\hat{\mathbf{R}}_{x x^{*}}(\alpha, \tau, N)$ to obtain

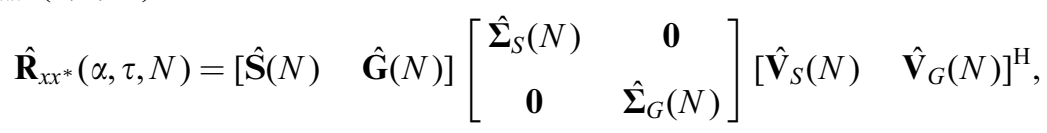

where $[\hat{\mathbf{S}}(N) \hat{\mathbf{G}}(N)]$ and $\left[\hat{\mathbf{V}}_{S}(N) \hat{\mathbf{V}}_{G}(N)\right]$ are two unitary matrices with the dimensions of $\hat{\mathbf{S}}(N)$ and $\hat{\mathbf{V}}_{S}(N)$ given by $M \times d$. On the other hand, the dimensions of $\hat{\mathbf{G}}(N)$ and $\hat{\mathbf{V}}_{G}(N)$ are $M \times(M-d)$. The diagonal elements of the diagonal matrices $\hat{\boldsymbol{\Sigma}}_{S}(N)$ and $\hat{\boldsymbol{\Sigma}}_{G}(N)$ are nonnegative and appear in decreasing order. Moreover, $\hat{\mathbf{\Sigma}}_{G}(N) \rightarrow 0$ as $N \rightarrow \infty$. Finally, find the $d$ minima of $\left\|\hat{\mathbf{G}}^{\mathrm{H}}(N) \mathbf{a}(\psi)\right\|^{2}$ or the $d$ maxima of $\left\|\hat{\mathbf{S}}^{\mathrm{H}}(N) \mathbf{a}(\psi)\right\|^{2}$, where $\mathbf{a}(\psi)$ are the directional vector corresponding to an impinging angle $\psi$ and $\|\cdot\|$ denotes the norm operator. The obtained $d$ values of $\psi$ are then taken as the DOA estimates of SOIs.

\section{Performance analysis in the presence of CFE}

Here, the performance of CC-MUSIC in the presence of CFE is evaluated. From (3), the sample conjugate cyclic correlation matrix $\hat{\mathbf{R}}_{x x^{*}}(f, \tau)$ is further expressed as

$$
\begin{aligned}
\hat{\mathbf{R}}_{x x^{*}}(f, \tau) & =\left\langle\mathbf{x}(t) \mathbf{x}^{\mathrm{T}}(t-\tau) \mathrm{e}^{-\mathrm{j} 2 \pi f t}\right\rangle_{T} \\
& \approx \sum_{m=1}^{d} \hat{r}_{s_{m} s_{m}^{*}}(f, \tau) \mathbf{a}\left(\theta_{m}\right) \mathbf{a}^{\mathrm{T}}\left(\theta_{m}\right)+\sum_{l=1}^{J} \hat{r}_{i_{l} i_{l}^{*}}(f, \tau) \mathbf{a}\left(\phi_{l}\right) \mathbf{a}^{\mathrm{T}}\left(\phi_{l}\right)+\hat{\mathbf{R}}_{n n^{*}}(f, \tau)
\end{aligned}
$$

when $T$ is large enough, where $\hat{r}_{a b^{*}}(f, \tau)$ denotes the sample conjugate cyclic correlation function of $a(t)$ and $b(t)$. We note from (5) that the cross terms disappear because of uncorrelation between the SOIs, the SNOIs, and noise. Due to the fact that the cyclic spectrum of a cyclostationary signal is discrete in the cycle frequency, the conjugate cyclic correlation functions of the $m$ th SOI and $l$ th SNOI are expressed as

$$
r_{S_{m} s_{m}^{*}}(f, \tau)=\sum_{n} \rho_{m, n}(\tau) \delta\left(f-\alpha_{n}\right) \quad \text { and } \quad r_{i_{l} i_{l}^{*}}(f, \tau)=\sum_{n} \varrho_{l, n}(\tau) \delta\left(f-\beta_{l, n}\right),
$$


respectively, where $\rho_{m, n}(\tau)$ and $\varrho_{l, n}(\tau)$ denote the strength of the $m$ th SOI and the $l$ th cyclostationary SNOI at cycle frequencies $\alpha_{n}$ and $\beta_{l, n}$, respectively. Using the properties of Fourier transform, the sample conjugate cyclic correlation function of the $m$ th SOI is given by

$$
\begin{aligned}
\hat{r}_{s_{m} s_{m}^{*}}(f, \tau) & =\left\langle s\left(t+\frac{\tau}{2}\right) s\left(t-\frac{\tau}{2}\right) \mathrm{e}^{-\mathrm{j} 2 \pi f t}\right\rangle_{T} \\
& =\frac{1}{T} \int_{-\infty}^{\infty} \operatorname{rect}\left(\frac{t}{T}\right) s\left(t+\frac{\tau}{2}\right) s\left(t-\frac{\tau}{2}\right) \mathrm{e}^{-\mathrm{j} 2 \pi f t} \mathrm{~d} t \\
& =\left[\int_{-\infty}^{\infty} \operatorname{rect}\left(\frac{t}{T}\right) \mathrm{e}^{-\mathrm{j} 2 \pi f t} \mathrm{~d} t\right] \otimes\left[\frac{1}{T} \int_{-\infty}^{\infty} \operatorname{rect}\left(\frac{t}{T}\right) s\left(t+\frac{\tau}{2}\right) s\left(t-\frac{\tau}{2}\right) \mathrm{e}^{-\mathrm{j} 2 \pi f t} \mathrm{~d} t\right] \\
& \approx \operatorname{TSINC}(f T) \otimes \sum_{n} \hat{\rho}_{m, n}(\tau) \delta\left(f-\alpha_{n}\right) \\
& =\sum_{n} \hat{\rho}_{m, n}(\tau) \operatorname{SINC}\left(\left(f-\alpha_{n}\right) T\right),
\end{aligned}
$$

where

$$
\operatorname{rect}\left(\frac{t}{T}\right)= \begin{cases}1 & \text { for }-\frac{T}{2} \leqslant t \leqslant \frac{T}{2} \\ 0 & \text { elsewhere }\end{cases}
$$

and

$$
\operatorname{SINC}(f T)=\frac{\sin (\pi f T)}{\pi f T}
$$

is the sinc function, and " $\otimes$ " denotes the convolution operation. Similarly, the sample conjugate cyclic correlation function of the $l$ th SNOI is given by

$$
\hat{r}_{i_{i} i_{l}^{*}}(f, \tau) \approx \sum_{n} \hat{\varrho}_{l, n}(\tau) \operatorname{SINC}\left(\left(f-\beta_{l, n}\right) T\right) .
$$

Substituting (7) and (10) into (5), we obtain

$$
\begin{aligned}
\hat{\mathbf{R}}_{x x^{*}}(f, \tau) \approx & \sum_{m=1}^{d}\left\{\sum_{n} \hat{\rho}_{m, n}(\tau) \operatorname{SINC}\left(\left(f-\alpha_{n}\right) T\right)\right\} \mathbf{a}\left(\theta_{m}\right) \mathbf{a}^{\mathrm{T}}\left(\theta_{m}\right) \\
& +\sum_{l=1}^{J}\left\{\sum_{n} \hat{\varrho}_{l, n}(\tau) \operatorname{SINC}\left(\left(f-\beta_{l, n}\right) T\right)\right\} \mathbf{a}\left(\phi_{l}\right) \mathbf{a}^{\mathrm{T}}\left(\phi_{l}\right)+\hat{\mathbf{R}}_{n n^{*}}(f, \tau) .
\end{aligned}
$$

For simplicity, we consider the case that there exists only one SOI, that is, $d=1$. Moreover, we assume $f=\alpha_{1}+\Delta \alpha$, where $\Delta \alpha$ represents the amount of CFE. From (11), we have

$$
\begin{aligned}
& \frac{\mathbf{a}^{\mathrm{H}}\left(\theta_{1}\right) \hat{\mathbf{R}}_{x x^{*}}\left(\alpha_{1}+\Delta \alpha, \tau\right) \mathbf{a}^{*}\left(\theta_{1}\right)}{M^{2}} \\
& \approx \hat{\rho}_{1,1}(\tau) \operatorname{SINC}(\Delta \alpha T)+\sum_{n \neq 1} \hat{\rho}_{1, n}(\tau) \operatorname{SINC}\left(\tilde{\alpha}_{n} T\right) \\
& \quad+\sum_{l=1}^{J}\left\{\sum_{n} \hat{\varrho}_{l, n}(\tau) \operatorname{SINC}\left(\tilde{\beta}_{l, n} T\right)\right\} \frac{\left|\mathbf{a}^{\mathrm{H}}\left(\theta_{1}\right) \mathbf{a}\left(\phi_{l}\right)\right|^{2}}{M^{2}}+\frac{\mathbf{a}^{\mathrm{H}}\left(\theta_{1}\right) \hat{\mathbf{R}}_{n n^{*}}\left(\alpha_{1}+\Delta \alpha, \tau\right) \mathbf{a}^{*}\left(\theta_{1}\right)}{M^{2}},
\end{aligned}
$$


where $\tilde{\alpha}_{n}=\alpha_{1}-\alpha_{n}+\Delta \alpha, \tilde{\beta}_{l, n}=\alpha_{1}-\beta_{l, n}+\Delta \alpha$, and $\mathbf{a}^{\mathrm{H}}\left(\theta_{1}\right) \mathbf{a}\left(\theta_{1}\right)=M$. Because $\mathbf{R}_{x x^{*}}(\alpha, \tau)=r_{s s^{*}}(\alpha, \tau) \mathbf{a}\left(\theta_{1}\right) \mathbf{a}^{\mathrm{T}}\left(\theta_{1}\right)$ when $d=1,(12)$ is an estimate of $\hat{r}_{s s^{*}}(f, \tau)$ and represents the dominance of the SOI in $\hat{\mathbf{R}}_{x x^{*}}(f, \tau)$. Consider the case where $\alpha_{n}$ 's and $\beta_{l, n}$ 's are well separated. Since the value of $\operatorname{SINC}(f T)$ is small enough for large $f T$, we note that (12) is approximately equal to $\hat{\rho}_{1,1}(\tau)$ which is the corresponding sample conjugate cyclic correlation function of the SOI at $\alpha_{1}$. On the other hand, if $\Delta \alpha \neq 0$, due to the fact that $\operatorname{SINC}(\Delta \alpha T)=0$ when $T=T_{k}= \pm k / \Delta \alpha, k=1,2, \ldots$, we note that the magnitude of (12) decreases and, hence, the estimation error of DOA increases as $T$ approaches $T_{k}$. Moreover, it would be expected from (12) that there exist periodic peaks in the curve of the estimation error versus the number of snapshots for direction finding using the CC-MUSIC under CFE.

\section{The proposed technique}

\subsection{Cycle frequency estimation method}

Consider a projection operator $\mathbf{P}=v \mathbf{S}_{s} \mathbf{S}_{s}^{\mathrm{H}}+(1-v)\left(\mathbf{I}-\mathbf{S}_{s} \mathbf{S}_{s}^{\mathrm{H}}\right)$, where $0.5<v \leqslant 1$, I is the identity matrix with appropriate size, and $\mathbf{S}_{s}$ spans the same subspace as that of $\left[\mathbf{a}\left(\theta_{1}\right), \ldots, \mathbf{a}\left(\theta_{d}\right)\right]$. Moreover, $\mathbf{S}_{s}^{\mathrm{H}} \mathbf{S}_{s}=\mathbf{I}$. Then, we form two vectors $\tilde{\mathbf{x}}(t)=\mathbf{P} \mathbf{x}(t)$ and $\tilde{\mathbf{u}}(t)=\tilde{\mathbf{x}}^{*}(t-\tau) \mathrm{e}^{\mathrm{j} 2 \pi f t}=\mathbf{P}^{*} \mathbf{x}^{*}(t-\tau) \mathrm{e}^{\mathrm{j} 2 \pi f t}=\mathbf{P}^{*} \mathbf{u}(t)$. The parameter $v$ is used to increase the components of $\tilde{\mathbf{x}}(t)$ and $\tilde{\mathbf{u}}(t)$ residing in the subspace of SOIs. Using (2), we construct a Hermitian matrix $\boldsymbol{\Psi}$ as

$$
\boldsymbol{\Psi}=\mathbf{R}_{\tilde{x} \tilde{u}} \mathbf{R}_{u u}^{-1} \mathbf{R}_{\tilde{x} \tilde{u}}^{\mathrm{H}}=\left(\mathbf{P R}_{x x^{*}}(f, \tau) \mathbf{P}^{\mathrm{T}}\right) \mathbf{R}_{u u}^{-1}\left(\mathbf{P}^{*} \mathbf{R}_{x x}^{\mathrm{H}}(f, \tau) \mathbf{P}^{\mathrm{H}}\right) .
$$

Since $\mathbf{P a}\left(\theta_{m}\right)=v \mathbf{a}\left(\theta_{m}\right)$, we have $\mathbf{a}^{\mathrm{T}}\left(\theta_{m}\right) \mathbf{P}^{\mathrm{T}} \mathbf{R}_{u u}^{-1} \mathbf{P}^{*} \mathbf{a}^{*}\left(\theta_{k}\right)=v^{2}\left(\mathbf{a}^{\mathrm{H}}\left(\theta_{m}\right) \mathbf{R}_{x x}^{-1} \mathbf{a}\left(\theta_{k}\right)\right)^{*} \approx 0$ if $m \neq k$. Moreover, due to uncorrelation between the SOIs, the SNOIs, and noise, we obtain $\mathbf{R}_{x x^{*}}(f, \tau)=\sum_{m=1}^{d} r_{s_{m} s_{m}^{*}}(f, \tau) \mathbf{a}\left(\theta_{m}\right) \mathbf{a}^{\mathrm{T}}\left(\theta_{m}\right)+$ $\sum_{l=1}^{J} r_{i_{l} i_{l}^{*}}(f, \tau) \mathbf{a}\left(\phi_{l}\right) \mathbf{a}^{\mathrm{T}}\left(\phi_{l}\right)$. Accordingly, it is easy to show that

$$
\begin{aligned}
\boldsymbol{\Psi} \approx & v^{4} \sum_{m=1}^{d}\left(\mathbf{a}^{\mathrm{T}}\left(\theta_{m}\right) \mathbf{R}_{u u}^{-1} \mathbf{a}^{*}\left(\theta_{m}\right)\right)\left|r_{s_{m} s_{m}^{*}}(f, \tau)\right|^{2} \mathbf{a}\left(\theta_{m}\right) \mathbf{a}^{\mathrm{H}}\left(\theta_{m}\right) \\
& +\sum_{l=1}^{J}\left(\mathbf{a}^{\mathrm{T}}\left(\phi_{l}\right) \mathbf{P}^{\mathrm{T}} \mathbf{R}_{u u}^{-1} \mathbf{P}^{*} \mathbf{a}^{*}\left(\phi_{l}\right)\right)\left|r_{i_{l} i_{l}^{*}}(f, \tau)\right|^{2}\left(\mathbf{P a}\left(\phi_{l}\right)\right)\left(\mathbf{P a}\left(\phi_{l}\right)\right)^{\mathrm{H}} \\
& + \text { Cross terms including the SNOIs. }
\end{aligned}
$$

Since $r_{i, i} i_{l}(\alpha, \tau)=0$, we note from (14) that $\Psi$ becomes a rank- $d$ matrix with the $d$ eigenvectors spanning the same subspace as that of $\left[\mathbf{a}\left(\theta_{1}\right), \ldots, \mathbf{a}\left(\theta_{d}\right)\right]$ when $f=\alpha$. For practical implementation, $\boldsymbol{\Psi}$ is computed by using $n$ data snapshots and given by

$$
\hat{\mathbf{\Psi}}(n)=\hat{\mathbf{R}}_{\tilde{x} \tilde{u}}(n) \hat{\mathbf{R}}_{u u}^{-1}(n) \hat{\mathbf{R}}_{\tilde{x} \tilde{u}}^{\mathrm{H}}(n),
$$

where $\hat{\mathbf{R}}_{u u}^{-1}(n)$ and $\hat{\mathbf{R}}_{\tilde{x} \tilde{u}}(n)$ represent the results computed by using $n$ data snapshots for $\mathbf{R}_{u u}^{-1}$ and $\mathbf{R}_{\tilde{x} \tilde{u}}$, respectively. Let the $m$ th largest eigenvalue of $\hat{\mathbf{\Psi}}(n)$ and the corresponding eigenvector be $\hat{\lambda}_{m}(n)$ and $\hat{\mathbf{e}}_{m}(n)$, respectively. Then, we set the associated projection operator to $\hat{\mathbf{P}}(n)=v \hat{\mathbf{S}}_{s}(n-1) \hat{\mathbf{S}}_{s}^{\mathrm{H}}(n-1)+(1-v)(\mathbf{I}-$ $\left.\hat{\mathbf{S}}_{s}(n-1) \hat{\mathbf{S}}_{s}^{\mathrm{H}}(n-1)\right)$, where $\hat{\mathbf{S}}_{s}(n-1)=\left[\hat{\mathbf{e}}_{1}(n-1), \ldots, \hat{\mathbf{e}}_{d}(n-1)\right]$. Accordingly, we have $\tilde{\mathbf{x}}(n)=\hat{\mathbf{P}}(n) \mathbf{x}(n)$ and $\tilde{\mathbf{u}}(n)=\hat{\mathbf{P}}^{*}(n) \mathbf{u}(n)=\tilde{\mathbf{x}}^{*}(n-\tau) \mathrm{e}^{\mathrm{j} 2 \pi \hat{\alpha}(n) n T_{s}}$, where $\hat{\alpha}(n)$ represents the estimate of the cycle frequency $\alpha$ obtained by using $n$ data snapshots. Consequently, $\hat{\mathbf{R}}_{\tilde{x} \tilde{u}}(n)$ can be computed from $\hat{\mathbf{R}}_{x u}(n)$ based on a recursive formula as follows:

$$
\hat{\mathbf{R}}_{\tilde{x} \tilde{u}}(n)=\hat{\mathbf{P}}(n) \hat{\mathbf{R}}_{x u}(n) \hat{\mathbf{P}}^{\mathrm{T}}(n)=\frac{n-1}{n} \hat{\mathbf{P}}(n) \hat{\mathbf{R}}_{x u}(n-1) \hat{\mathbf{P}}^{\mathrm{T}}(n)+\frac{1}{n} \tilde{\mathbf{x}}(n) \tilde{\mathbf{u}}^{\mathrm{H}}(n),
$$


where $\hat{\mathbf{R}}_{x u}(n)=1 / n \sum_{i=1}^{n} \mathbf{x}(i) \mathbf{u}^{\mathrm{H}}(i)$ represents the sample cross-correlation matrix computed by using $n$ data snapshots for $\mathbf{x}(t)$ and $\mathbf{u}(t), \mathbf{x}(i)=\mathbf{x}\left(i T_{s}\right)$ with sampling interval $=T_{s}$. By using the eigenspace of $\hat{\mathbf{\Psi}}(n-1)$ and the new received array data $\mathbf{x}(n)$, an appropriate estimate of the cycle frequency regarding the SOI is obtained as follows: Substituting (16) into (15) and performing some algebraic manipulations, we obtain

$$
\begin{aligned}
\hat{\mathbf{e}}_{m}^{\mathrm{H}}(n-1) \hat{\mathbf{\Psi}}(n) \hat{\mathbf{e}}_{m}(n-1) \\
=\hat{\mathbf{e}}_{m}^{\mathrm{H}}(n-1) \hat{\mathbf{R}}_{\tilde{x} \tilde{u}}(n) \hat{\mathbf{R}}_{u u}^{-1}(n) \hat{\mathbf{R}}_{\tilde{x} \tilde{u}}^{\mathrm{H}}(n) \hat{\mathbf{e}}_{m}(n-1) \\
=\left(\frac{n-1}{n}\right)^{2} \hat{\mathbf{e}}_{m}^{\mathrm{H}}(n-1) \hat{\mathbf{P}}(n) \hat{\mathbf{R}}_{x u}(n-1) \hat{\mathbf{P}}^{\mathrm{T}}(n) \hat{\mathbf{R}}_{u u}^{-1}(n) \hat{\mathbf{P}}^{*}(n) \hat{\mathbf{R}}_{x u}^{\mathrm{H}}(n-1) \hat{\mathbf{P}}^{\mathrm{H}}(n) \hat{\mathbf{e}}_{m}(n-1) \\
\quad+\left(\frac{1}{n^{2}}\right)\left\{\tilde{\mathbf{x}}^{\mathrm{T}}(n-\tau) \hat{\mathbf{R}}_{u u}^{-1}(n) \tilde{\mathbf{x}}^{*}(n-\tau)\right\}\left|\hat{\mathbf{e}}_{m}^{\mathrm{H}}(n-1) \tilde{\mathbf{x}}^{\mathrm{H}}(n)\right|^{2} \\
\quad+\left(\frac{n-1}{n^{2}}\right) \hat{\mathbf{e}}_{m}^{\mathrm{H}}(n-1) \hat{\mathbf{P}}(n) \hat{\mathbf{R}}_{x u}(n-1) \hat{\mathbf{P}}^{\mathrm{T}}(n) \hat{\mathbf{R}}_{u u}^{-1}(n) \tilde{\mathbf{x}}^{*}(n-\tau) \tilde{\mathbf{x}}^{\mathrm{H}}(n) \hat{\mathbf{e}}_{m}(n-1) \mathrm{e}^{\mathrm{j} 2 \pi \hat{\alpha}(n) n T_{s}} \\
\quad+\left(\frac{n-1}{n^{2}}\right) \hat{\mathbf{e}}_{m}^{\mathrm{H}}(n-1) \tilde{\mathbf{x}}(n) \tilde{\mathbf{x}}^{\mathrm{T}}(n-\tau) \hat{\mathbf{R}}_{u u}^{-1}(n) \hat{\mathbf{P}}^{*}(n) \hat{\mathbf{R}}_{x u}^{\mathrm{H}}(n-1) \hat{\mathbf{P}}^{\mathrm{H}}(n) \hat{\mathbf{e}}_{m}(n-1) \mathrm{e}^{-\mathrm{j} 2 \pi \hat{\alpha}(n) n T_{s}} .
\end{aligned}
$$

Let $n$ be large enough such that $\hat{\mathbf{R}}_{u u}^{-1}(n) \cong \mathbf{R}_{u u}^{-1}(n-1), \hat{\mathbf{P}}(n) \cong \hat{\mathbf{P}}(n-1)$, and $\hat{\mathbf{e}}_{m}^{\mathrm{H}}(n-1) \hat{\mathbf{\Psi}}(n) \hat{\mathbf{e}}_{m}(n-1) \cong \hat{\lambda}_{m}(n-1)$ under the assumption of ergodicity for the received array data. Substituting these three approximations into (17) and performing some algebraic manipulations yields

$$
\begin{aligned}
\underbrace{(2 n-1) \hat{\lambda}_{m}(n-1)-\left\{\tilde{\mathbf{x}}^{\mathrm{T}}(n-\tau) \hat{\mathbf{R}}_{u u}^{-1}(n-1) \tilde{\mathbf{x}}^{*}(n-\tau)\right\}\left|\hat{\mathbf{e}}_{m}^{\mathrm{H}}(n-1) \tilde{\mathbf{x}}(n)\right|^{2}}_{b_{m}(n)} \\
=(n-1)\{\underbrace{\left(\hat{\mathbf{e}}_{m}^{\mathrm{H}}(n-1) \hat{\mathbf{P}}(n) \hat{\mathbf{R}}_{x u}(n-1) \hat{\mathbf{P}}^{\mathrm{T}}(n) \hat{\mathbf{R}}_{u u}^{-1}(n-1) \tilde{\mathbf{x}}^{*}(n-\tau)\right)\left(\tilde{\mathbf{x}}^{\mathrm{H}}(n) \hat{\mathbf{e}}_{m}(n-1)\right)}_{a_{m}(n)} \mathrm{e}^{\mathrm{j} 2 \pi \hat{\alpha}(n) n T_{s}} \\
\quad+\underbrace{\left(\hat{\mathbf{e}}_{m}^{\mathrm{H}}(n-1) \hat{\mathbf{P}}(n) \hat{\mathbf{R}}_{x u}(n-1) \hat{\mathbf{P}}^{\mathrm{T}}(n) \hat{\mathbf{R}}_{u u}^{-1}(n-1) \tilde{\mathbf{x}}^{*}(n-\tau)\right)^{*}\left(\tilde{\mathbf{x}}^{\mathrm{H}}(n) \hat{\mathbf{e}}_{m}(n-1)\right)^{*}}_{a_{m}^{*}(n)} \mathrm{e}^{\left.-\mathrm{j} 2 \pi \hat{\alpha}(n) n T_{s}\right\},}
\end{aligned}
$$

where the term represented by $b_{m}(n)$ is a real number, while the term represented by $a_{m}(n)$ is a complex one. From (18), we obtain

$$
\left\{(n-1) a_{m}(n)\right\} v^{2}(n)-\left\{b_{m}(n)\right\} v(n)+(n-1) a_{m}^{*}(n)=0
$$

for $m=1,2, \ldots, d$, where $v(n)=\mathrm{e}^{\mathrm{j} 2 \pi \hat{\alpha}(n) n T_{s}}$. Eq. (19) reveals that

$$
v(n)=\frac{b(n) \pm \sqrt{b^{2}(n)-4(n-1)^{2}|a(n)|^{2}}}{2(n-1) a(n)},
$$

where $a(n)=1 / d \sum_{m=1}^{d} a_{m}(n)$ and $b(n)=\frac{1}{d} \sum_{m=1}^{d} b_{m}(n)$. Therefore, we have the following equation for estimating the cycle frequency $\alpha$

$$
\hat{\alpha}(n)=\frac{\angle v(n)+2 \pi n \kappa}{2 \pi n T_{s}}=\frac{1}{T_{s}}\left(\kappa-\frac{1}{2 \pi n} \angle a(n)\right),
$$


where $\angle x$ denotes the principle value of phase for complex number $x$ and $\kappa$ is chosen such that $\mid \hat{\alpha}(n)-$ $\hat{\alpha}(n-1) \mid<1 / T_{s}$. In order to reduce the effect of finite data samples on the three approximations, namely, $\hat{\mathbf{R}}_{u u}^{-1}(n) \cong \hat{\mathbf{R}}_{u u}^{-1}(n-1), \hat{\mathbf{P}}(n) \cong \hat{\mathbf{P}}(n-1)$, and $\hat{\mathbf{e}}_{m}^{\mathrm{H}}(n-1) \hat{\mathbf{\Psi}}(n) \hat{\mathbf{e}}_{m}(n-1) \cong \hat{\lambda}_{m}(n-1)$ made for deriving (18), we refine the estimated cycle frequency as follows: First, we compute $\hat{\alpha}(n, l)=\hat{\alpha}(n)+\left(l / 2 n T_{s}\right)$, where the integer $l \in\{-1,0,1\}$. Then, we update $\hat{\mathbf{R}}(n, l)$ during the estimation procedure by utilizing the following recursive formula

$$
\hat{\mathbf{R}}(n, l)= \begin{cases}\frac{1}{n} \sum_{i=1}^{n} \mathbf{x}(i) \mathbf{x}^{\mathrm{T}}(i-\tau) \mathrm{e}^{-\mathrm{j} 2 \pi \hat{\alpha}(n, l) i T_{s}}, & \text { for } n \leqslant L_{b}, \\ \frac{n-1}{n} \hat{\mathbf{R}}_{x u}(n-1)+\frac{1}{n} \sum_{i=n-L_{b}+1}^{n} \mathbf{x}(i) \mathbf{x}^{\mathrm{T}}(i-\tau) \mathrm{e}^{-\mathrm{j} 2 \pi \hat{\alpha}(n, l) i T s}, & \text { for } n>L_{b}\end{cases}
$$

to save the computing cost, where $L_{b}$ is a preset positive integer and denotes the number of data snapshots used to update the corresponding correlations for heightening the effect of $\hat{\alpha}(n, l)$. We note from (22) that since the estimate of cycle frequency should be more and more accurate, $L_{b}$ data samples are used to enhance the corresponding component in $\hat{\mathbf{R}}(n, l)$. Based on (22), the value of $l$ which maximizes the largest eigenvalue of $\hat{\mathbf{P}}(n) \hat{\mathbf{R}}(n, l) \hat{\mathbf{P}}^{\mathrm{T}}(n) \hat{\mathbf{P}}^{*}(n) \hat{\mathbf{R}}^{\mathrm{H}}(n, l) \hat{\mathbf{P}}^{\mathrm{H}}(n)$ is selected. The corresponding $\hat{\alpha}(n, l)$ is then used as the desired estimate $\hat{\alpha}(n)$ of the cycle frequency $\alpha$ after receiving $n$ data snapshots. Moreover, we set $\hat{\mathbf{R}}_{x u}(n)$ to the corresponding $\hat{\mathbf{R}}(n, l)$.

\subsection{The robust direction finding}

After obtaining $\hat{\alpha}(n)$, we summarize the proposed technique step-by-step as follows:

Step 1: In the initial step, we set robust interval $=\Delta \alpha_{b}, n=N_{i}=1 /\left(2 \Delta \alpha_{b} T_{s}\right)$, the presumed cycle frequency $\hat{\alpha}\left(N_{i}\right)$, and $\hat{\mathbf{P}}\left(N_{i}\right)=\mathbf{I}$. Then, we compute the matrices $\hat{\mathbf{R}}_{x u}\left(N_{i}\right)$ and $\hat{\mathbf{R}}_{x x}^{-1}\left(N_{i}\right)$ by

$$
\hat{\mathbf{R}}_{x u}\left(N_{i}\right)=\frac{1}{N_{i}} \sum_{i=1}^{N_{i}} \mathbf{x}(i) \mathbf{x}^{\mathrm{T}}(i-\tau) \mathrm{e}^{-\mathrm{j} 2 \pi \hat{\alpha}\left(N_{i}\right) i T_{s}} \quad \text { and } \quad \hat{\mathbf{R}}_{x x}^{-1}\left(N_{i}\right)=\left\{\delta^{2} \mathbf{I}+\frac{1}{N_{i}} \sum_{i=1}^{N_{i}} \mathbf{x}(i) \mathbf{x}^{\mathrm{H}}(i)\right\}^{-1},
$$

where $\delta^{2}$ is a small constant and $\mathbf{I}$ denotes the identity matrix with appropriate size.

Step 2: Compute $\hat{\mathbf{B}}\left(N_{i}\right)=\hat{\mathbf{R}}_{\tilde{x} \tilde{u}}\left(N_{i}\right) \hat{\mathbf{R}}_{\tilde{x} \tilde{u}}^{\mathrm{H}}\left(N_{i}\right)$. Then, the FSD algorithm presented in [14] is used to obtain $\hat{\mathbf{e}}_{i}\left(N_{i}\right)$ for $i=1,2, \ldots, d$ as follows, where $\hat{\mathbf{e}}_{i}\left(N_{i}\right)$ denotes the eigenvector of $\hat{\mathbf{B}}\left(N_{i}\right)$ corresponding to the $i$ th largest eigenvalue.

(2.1) Generate an initial vector $\hat{\mathbf{r}}_{0}\left(N_{i}\right)$ as

$$
\hat{\mathbf{r}}_{0}\left(N_{i}\right)=\frac{\mathbf{g}}{\|\mathbf{g}\|}
$$

where $\mathbf{g}=\hat{\mathbf{B}}\left(N_{i}\right)[11 \cdots 1]^{\mathrm{T}}$ and $\|\mathbf{x}\|$ denotes the norm of the vector $\mathbf{x}$. Utilizing the Lanczos algorithm described in [14], we then compute

$$
\mathbf{q}_{1}=\hat{\mathbf{r}}_{0}\left(N_{i}\right) \quad \text { and } \quad \mathbf{q}_{k}=\frac{\hat{\mathbf{B}}\left(N_{i}\right) \mathbf{q}_{k-1}-a_{k-1} \mathbf{q}_{k-1}-b_{k-1} \mathbf{q}_{k-2}}{b_{k}} \quad \text { for } k=2, \ldots, d+1,
$$

where $a_{k}=\mathbf{q}_{k}^{\mathrm{H}} \hat{\mathbf{B}}\left(N_{i}\right) \mathbf{q}_{k}$ and $b_{k}=\left\|\mathbf{q}_{k}\right\|$. 
(2.2) Form the matrix $\mathbf{Q}=\left[\mathbf{q}_{1}, \mathbf{q}_{2}, \ldots, \mathbf{q}_{d+1}\right]$ and then compute $\mathbf{Q}^{\mathrm{H}} \hat{\mathbf{B}}\left(N_{i}\right) \mathbf{Q}$. It is easy to show that the result can be expressed as

$$
\mathbf{Q}^{\mathrm{H}} \hat{\mathbf{B}}\left(N_{i}\right) \mathbf{Q}=\left[\begin{array}{ccccccc}
a_{1} & b_{1} & 0 & \ldots & \ldots & \ldots & 0 \\
b_{1} & a_{2} & b_{2} & 0 & \ldots & \ldots & 0 \\
0 & b_{2} & a_{3} & b_{3} & 0 & \ldots & 0 \\
\ldots & \ldots & \ldots & \ldots & \ldots & \ldots & \ldots \\
0 & \ldots & \ldots & \ldots & \ldots & b_{d} & a_{d+1}
\end{array}\right] .
$$

(2.3) Compute $\hat{\mathbf{e}}_{i}\left(N_{i}\right)=\mathbf{Q} \hat{\mathbf{v}}_{i}$, where $\hat{\mathbf{v}}_{i}$ denotes the eigenvector of $\mathbf{Q}^{\mathrm{H}} \hat{\mathbf{B}}\left(N_{i}\right) \mathbf{Q}$ corresponding to the $i$ th largest eigenvalue.

Step 3: Construct $\hat{\mathbf{S}}_{s}(n)=\left[\hat{\mathbf{e}}_{1}(n), \ldots, \hat{\mathbf{e}}_{d}(n)\right]$ and compute the values of $\Omega(\psi)=1 / \mathbf{a}^{\mathrm{H}}(\psi)\left\{\mathbf{I}-\hat{\mathbf{S}}_{s}(n) \hat{\mathbf{S}}_{s}^{\mathrm{H}}(n)\right\} \mathbf{a}(\psi)$, where $\mathbf{a}(\psi)$ denotes the directional vector corresponding to the angle $\psi$. The $d$ maxima of $\Omega(\psi)$ are taken as the estimates of the angles of the $d$ SOIs.

Step 4: If a new snapshot $\mathbf{x}(n+1)$ is available, then go to Step 5. Otherwise, stop the process.

Step 5: Set $n=n+1$ and compute $\hat{\mathbf{R}}_{x x}^{-1}(n)=n /(n-1)\left\{\hat{\mathbf{R}}_{x x}^{-1}(n-1)-\left(\hat{\mathbf{R}}_{x x}^{-1}(n-1) \mathbf{x}(n) \mathbf{x}^{\mathrm{H}}(n) \hat{\mathbf{R}}_{x x}^{-1}(n-1) /\right.\right.$ $\left.\left.1+\mathbf{x}^{\mathrm{H}}(n) \hat{\mathbf{R}}_{x x}^{-1}(n-1) \mathbf{x}(n)\right)\right\}$ which is derived from the recursion of $\hat{\mathbf{R}}_{x x}(n)$ and the generalized inverse matrix lemma.

Step 6: Set $\hat{\mathbf{P}}(n)=v \hat{\mathbf{S}}_{s}(n-1) \hat{\mathbf{S}}_{s}^{\mathrm{H}}(n-1)+(1-v)\left(\mathbf{I}-\hat{\mathbf{S}}_{s}(n-1) \hat{\mathbf{S}}_{s}^{\mathrm{H}}(n-1)\right)$ and calculate $a(n), b(n), v(n)$, and, hence, $\hat{\alpha}(n)$ by from (18) to $(21)$.

Step 7: Compute $\hat{\alpha}(n, l)=\hat{\alpha}(n)+l / 2 n T_{s}$ for $l=-1,0$, and 1. If none of $\hat{\alpha}(n, l)$ satisfies $\left|\hat{\alpha}(n, l)-\hat{\alpha}\left(N_{i}\right)\right| \leqslant \Delta \alpha_{b}$, we set $\hat{\alpha}(n)=\hat{\alpha}(n-1)$ and $\hat{\mathbf{S}}_{s}(n)=\hat{\mathbf{S}}_{s}(n-1)$. Then, go to Step 3. Otherwise, we update $\hat{\mathbf{R}}(n, l)$ from (22) and perform the FSD algorithm on $\hat{\mathbf{P}}(n) \hat{\mathbf{R}}(n, l) \hat{\mathbf{P}}^{\mathrm{T}}(n) \hat{\mathbf{P}}^{*}(n) \hat{\mathbf{R}}^{\mathrm{H}}(n, l) \hat{\mathbf{P}}^{\mathrm{H}}(n)$ with the initial vector set to $\hat{\mathbf{r}}_{0}(n)=\hat{\mathbf{e}}_{1}(n-1)$ to find the associated $d$ largest eigenvalues and the $d$ corresponding eigenvectors. Accordingly, choose the $l$ which maximizes the largest eigenvalue and set the estimate $\hat{\alpha}(n)$ to the corresponding $\hat{\alpha}(n, l)$. Moreover, set $\hat{\mathbf{R}}_{x u}(n)=\hat{\mathbf{R}}(n, l)$ and the $d$ columns of $\hat{\mathbf{S}}_{s}(n)$ equal to the $d$ corresponding eigenvectors. Go to Step 3.

\subsection{Determination of the Robust Interval $\Delta \alpha_{b}$}

Here, an experimental manner to determine the robust interval $\Delta \alpha_{b}$ is presented. From (11), we note that there exist $\mathrm{SINC}_{d}$ functions which have mainlobes centered at $\alpha_{n}$ and $\beta_{l, n}$. Hence, after increasing the number of snapshots to such an $N_{b}$ that the mainlobes of these $\mathrm{SINC}_{d}$ functions become distinguishable from the sidelobes, we perform the following procedure to determine an appropriate robust interval $\Delta \alpha_{b}$.

Step 1: Compute $\left|\hat{r}_{x_{i} x_{i}^{*}}\left(f, 0, N_{b}\right)\right|, i=1,2, \ldots, M$, from $f=0$ to $1 / T_{s}$ and take the average given by $\xi(f)=$ $1 / M \sum_{i=1}^{M}\left|\hat{r}_{x_{i} x_{i}^{*}}\left(f, 0, N_{b}\right)\right|$, where $x_{i}$ denotes the data received by the $i$ th sensor element and $M$ is the number of array elements.

Step 2: Find two mainlobes which are the nearest and next nearest to $\hat{\alpha}\left(N_{i}\right)$ from $\xi(f)$. The widths of these mainlobes should be about twice of those of other sidelobes. Let these two mainlobes be centered at $f_{1}$ and $f_{2}$, respectively.

Step 3: Set $\Delta \alpha_{b}=\left|f_{1}-f_{2}\right| / 2(\gamma+1)$, where the integer $\gamma \geqslant 1$ is employed to reduce the effect of strong SNOI. In general, $\gamma$ is increased as the power of the SNOI increases.

Assume that the cycle frequencies of the $J$ SNOIs satisfy the condition of $\left|\beta_{J}\right|>\left|\beta_{J-1}\right|>\cdots>\left|\beta_{1}\right|>|\alpha|$ $>0$, where $\beta_{l}$ denotes the cycle frequency of the $l$ th SNOI and $\alpha$ the cycle frequency of the SOI. Moreover, 
the power of the $l$ th SNOI is comparable with that of SOI, while the powers of the first $(l-1)$ SNOIs are negligible in comparison with that of SOI. Based on the steps described above, some SNOIs may have cycle frequencies residing in the region of $\left\{\hat{\alpha}\left(N_{i}\right)-\Delta \alpha_{b}, \hat{\alpha}\left(N_{i}\right)+\Delta \alpha_{b}\right\}$. However, they have little effect on the proposed technique because of their low powers as compared to the SOIs.

\section{Computer simulations}

Consider a uniform linear array (ULA) with number of elements $M=8$ and interelement spacing $=$ half wavelength of SOI for all simulation examples. The sampling interval $T_{s}=0.2$ and $\tau=0$ are used. The noise is spatially white Gaussian with mean zero and variance one. For the proposed technique, $L_{b}$ in $(22), \delta^{2}$ in (23), $v$ in Step 6 of Section 4.2 are set to 50, 10 $0^{-6}$, and 0.9, respectively. The results of using the CC-MUSIC are also provided for comparison. All the simulation results are obtained by averaging 50 independent runs.
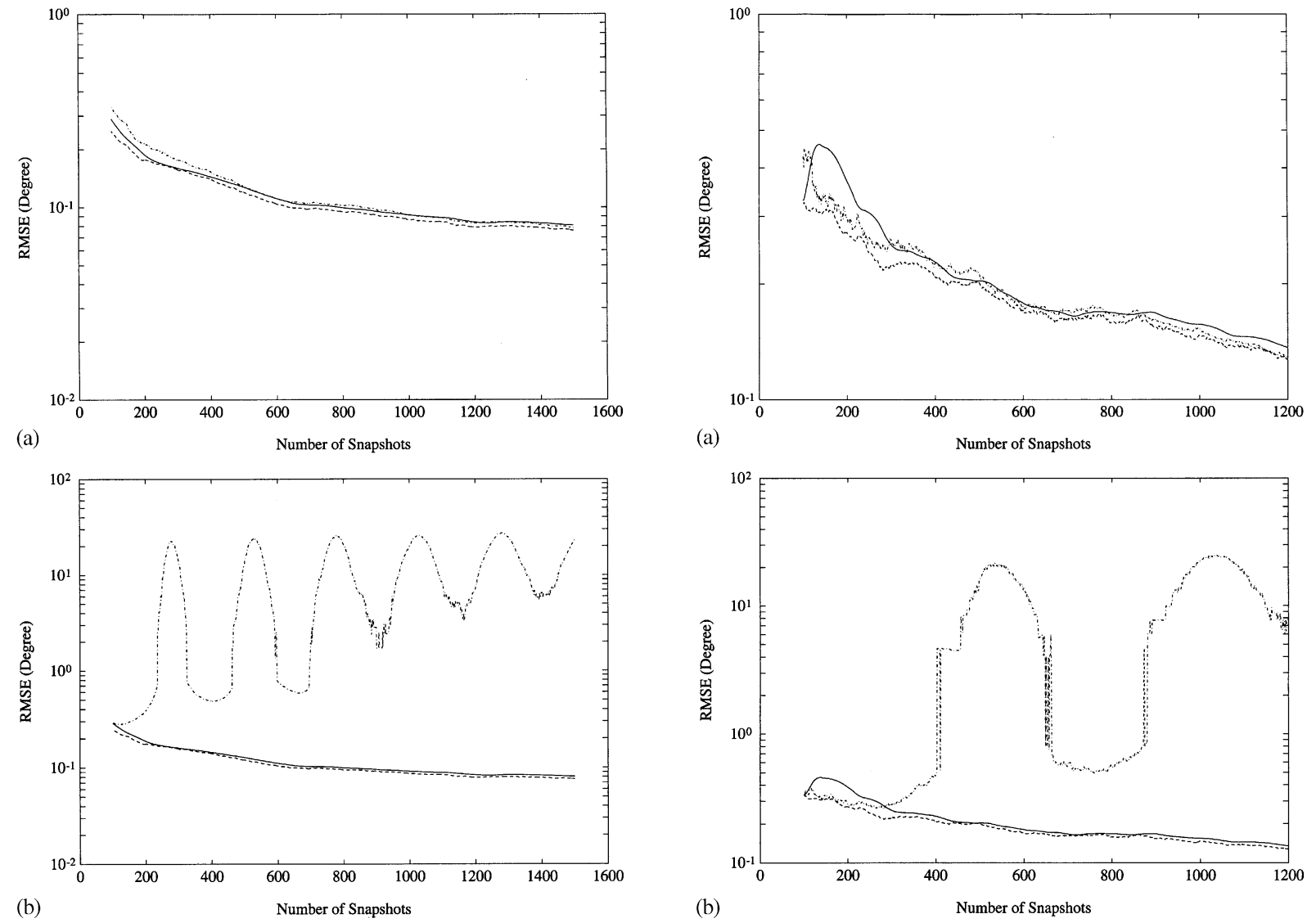

Fig. 1. The RMSE versus the number of snapshots for Example 1. (a) $\hat{\alpha}\left(N_{i}\right)=2.0$, (b) $\hat{\alpha}\left(N_{i}\right)=2.02$. — : The proposed method with $L_{b}=50$. ----: The proposed method with $L_{b} \rightarrow \infty$. $-\cdot-\cdot$ SVD-based conjugate Cyclic MUSIC.

Fig. 2. The RMSE versus the number of snapshots for Example 2. (a) $\hat{\alpha}\left(N_{i}\right)=1.0$, (b) $\hat{\alpha}\left(N_{i}\right)=1.01$. —: The proposed method with $L_{b}=50$. ---- : The proposed method with $L_{b} \rightarrow \infty$. $-\cdot-\cdot$ SVD-based conjugate Cyclic MUSIC. 
Example 1. In this case, a SOI impinging on the array from $5^{\circ}$ off broadside is a rectangular pulse shaped BPSK signal with carrier frequency $=1$, baud rate $=1$, and SNR $=0 \mathrm{~dB}$. A SNOI impinging from $-30^{\circ}$ off broadside is also a rectangular pulse shaped BPSK signal with carrier frequency $=1.5$, baud rate $=1$, and $\mathrm{SNR}=0 \mathrm{~dB}$. In addition, another SNOI impinging from $30^{\circ}$ off broadside is a DSB-AM signal with carrier frequency $=0.5$ and $\mathrm{SNR}=0 \mathrm{~dB} . N_{b}=25$ and $\gamma=1$ are used to get $\Delta \alpha_{b}=0.125$ from the procedure presented in Section 3. The results of the proposed technique with $L_{b} \rightarrow \infty$ are also provided for comparison. Fig. 1 plots the RMSE (root mean-square error) of the estimated DOA versus the number of snapshots under $\hat{\alpha}\left(N_{i}\right)=2$ and 2.02, respectively. From Fig. 1(a), we observe that the RMSE obtained by the proposed technique with $L_{b} \rightarrow \infty$ is smaller than that of using the CC-MUSIC when $\hat{\alpha}\left(N_{i}\right)=2$. Moreover, the RMSE obtained by the proposed technique with $L_{b}=50$ is almost comparable to that of using the CC-MUSIC. From Fig. 1(b), however, the proposed technique outperforms the CC-MUSIC in the presence of CFE. When $\hat{\alpha}\left(N_{i}\right)=2.02$, the RMSE has peaks at about $n=250 k, k=1,2, \ldots$ for the CC-MUSIC as expected by the theoretical analysis presented in Section 3.

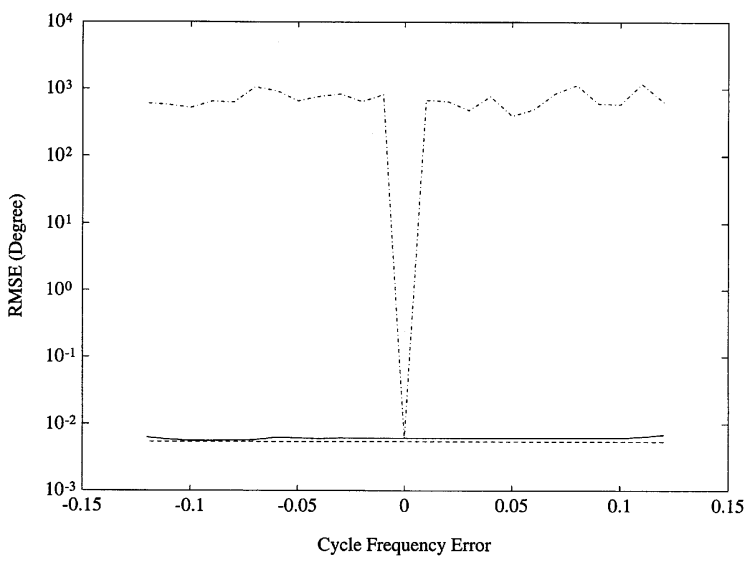

Fig. 3. The RMSE as a function of cycle frequency error for Example 3. — : The proposed method with $L_{b}=50$. $----:$ The proposed method with $L_{b} \rightarrow \infty .-\cdot-\cdot$ SVD-based conjugate Cyclic MUSIC.
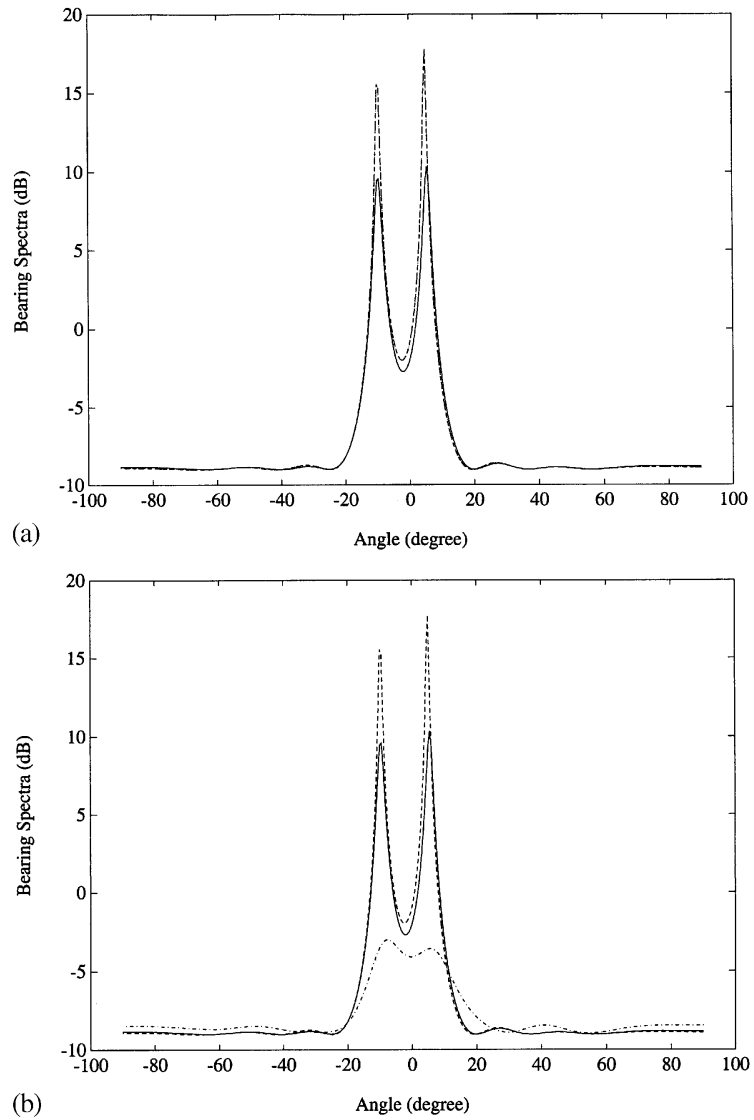

Fig. 4. The bearing spectra versus the impinging angle for Example 4. (a) $\hat{\alpha}\left(N_{i}\right)=2.0$, (b) $\hat{\alpha}\left(N_{i}\right)=2.02$. — : The proposed method with $L_{b}=50$. ---- : The proposed method with $L_{b} \rightarrow \infty$. $-\cdot-\cdot$ SVD-based conjugate Cyclic MUSIC. 
Example 2. In this case, a SOI impinging on the array from $5^{\circ}$ off broadside is a PAM signal with carrier frequency $=1$, baud rate $=1$, and $\mathrm{SNR}=6 \mathrm{~dB}$. Moreover, two SNOIs impinging on the array from $30^{\circ}$ and $40^{\circ}$

off broadside are also PAM signals with carrier frequencies $=1.5$ and 2 , baud rates $=\frac{5}{3}$ and $\frac{5}{3}$, and $\mathrm{SNR}=6$ and $6 \mathrm{~dB}$, respectively. $N_{b}=30$ and $\gamma=3$ are used to get $\Delta \alpha_{n}=0.042$ for this example. Fig. 2 depicts the RMSE of the estimated DOA versus the number of snapshots under $\alpha\left(N_{i}\right)=1$ and 1.01, respectively. We observe from Fig. 2 that the proposed technique with $L_{b}=50$ or $L_{b} \rightarrow \infty$ can provide the satisfactory results no matter whether the CFE exists or not. However, from Fig. 2(b), we note that the CC-MUSIC suffers from performance degradation in the presence of CFE and has severe RMSE at about $500 k, k=1,2, \ldots$, as expected.

Example 3. In this case, the SOI and the SNOIs are the same as those used in Example 1. 1000 data snapshots are used to obtained the simulation results. Fig. 3 depicts the RMSE of the estimated DOA versus the CFE regarding the SOI. From the figure, we observe that the RMSE of using the proposed technique almost equals a constant irrespective of CFE.

Example 4. In this example, two SOIs impinging from $5^{\circ}$ and $-10^{\circ}$ off broadside are rectangular pulse shaped BPSK signals with carrier frequencies $=1$ and 1 , baud rates $=1$ and 1 , and $\mathrm{SNR}=0$ and $0 \mathrm{~dB}$, respectively. A SNOI impinging from $30^{\circ}$ off broadside is also a rectangular pulse shaped BPSK signal with carrier frequency $=0.5$, baud rate $=1$, and $\mathrm{SNR}=0 \mathrm{~dB}$. Fig. 4 plots the resulting bearing spectra which are obtained by using 500 data snapshots under $\hat{\alpha}\left(N_{i}\right)=2$ and 2.02, respectively. From Fig. 4(a), we observe that when the actual cycle frequency is exactly known and $L_{b}$ approaches infinite, the proposed technique possesses almost the same performance as the CC-MUSIC. Moreover, the proposed technique possesses satisfactory performance when $L_{b}=50$ is used. From Fig. 4(b), we observe that the proposed technique can effectively alleviate the performance degradation of using the CC-MUSIC in the presence of CFE.

\section{Conclusion}

This paper has presented a robust technique for direction finding using cyclostationary signals with cycle frequency error (CFE). The performance degradation of the CC-MUSIC due to CFE has been analyzed. Based on the theoretical results, an efficient technique has been developed to cope with the performance deterioration of the CC-MUSIC due to CFE. According to the proposed technique, an efficient procedure exploits the inherent eigenstructure of cyclic conjugate correlation matrix to estimate cycle frequency. The estimation procedure is then incorporated with a fast subspace decomposition algorithm to deal with direction finding against CFE. As compared to the CC-MUSIC, computer simulations have shown that the proposed technique can effectively cure the performance deterioration due to CFE.

\section{References}

[1] T.E. Biedka, B.G. Agee, Subinterval cyclic MUSIC - robust DF with error in cycle frequency knowledge, Proceedings of the 25th Asilomar Conference Signals, Systems and Computers, Pacific Grove, CA, November 1991, pp. 262-266.

[2] R.D. DeGroat, R.A. Roberts, Efficient, numerically stabilized rank-one eigenstructure updating, IEEE Trans. Acoust. Speech Signal Process. 38 (February 1990) 301-316.

[3] W.A. Gardner, Spectral correlation of modulated signals: Part I-analog modulation, IEEE Trans. Commun. COM-35 (June 1987) 584-594.

[4] W.A. Gardner, Simplification of MUSIC and ESPRIT by exploitation of cyclostationarity, Proc. IEEE 76 (July 1988 ) $845-847$.

[5] W.A. Gardner, Exploitation of spectral redundancy in cyclostationary signals, IEEE SP Mag. (April 1991) 14-36.

[6] W.A. Gardner, Cyclostationarity in Communications and Signal Processing, IEEE Press, New York, 1994. 
[7] W.A. Gardner, W.A. Brown III, C.-K. Chen, Spectral correlation of modulated signals: Part II-digital modulation, IEEE Trans. Commun. COM-35 (June 1987) 595-601.

[8] F. Gini, G.B. Giannakis, Frequency offset and symbol timing recovery in flat-fading channels: a cyclostationary approach, IEEE Trans. Commun. 46 (March 1998) 400-411.

[9] S.V. Schell, Performance analysis of the cyclic MUSIC method of direction estimation for cyclostationary signals, IEEE Trans. Signal Process. 42 (November 1994) 3043-3050.

[10] S.V. Schell, W.A. Gardner, Progress on signal-selective direction finding, Proceedings of the 5th ASSP Workshop on Spectrum Estimation and Modeling, Rochester, NY, October 1990, pp. 144-148.

[11] S.V. Schell, W.A. Gardner, Cramér-Rao lower bound for directions of arrival of Gaussian cyclostationary signals, IEEE Trans. Inform. Theory 38 (July 1992) 1418-1422.

[12] R.O. Schmidt, Multiple emitter location and signal parameter estimation, IEEE Trans. Antennas Propagation AP-34 (March 1986) $276-280$

[13] E. Serpedin, A. Chevreuil, G.B. Giannakis, P. Loubaton, Blind channel and carrier frequency offset estimation using periodic modulation precoders, IEEE Trans. Signal Process. 48 (August 2000) 2389-2405.

[14] G. Xu, T. Kailath, Fast subspace decomposition, IEEE Trans. Signal Process. 42 (March 1994) 539-551. 